\title{
TOWARDS NEW WEB APPLICATION DEVELOPMENT PRACTICES
}

\author{
Angeliki POUL YMENAKOU ${ }^{1}$ William DRAKOS ${ }^{2}$, Anastasia PAPAZAFEIROPOULOU ${ }^{3}$, Georgios DOUKIDIS ${ }^{4}$ \\ HEITRUN - Hellenic ELectronic Trading Research UNit \\ Athens University of Economics and Business, 76 Patission st 10434 Athens Greece \\ http://heltrun.aueb.gr \\ E-mails: 'akp@ aueb.gr, ${ }^{2}$ asd@ aueb.gr. 3 agp@ aueb.gr, ${ }^{4}$ gjd@ aueb.gr
}

\begin{abstract}
Electronic Commerce over the Intemet, aims to become a global conveyor belt of business transactions. Web applications of increasing sophistication emerge in almost every business sector, reflecting a variety of technical and technological approaches. In this paper we argue that system developers need to reconsider their professional practices in the context of these new technologies by taking advantage of opportunities like short response cycles and easy diffusion of systems results, while they recognise the limitations of traditional practice. We discuss a framework of IS development issues for Internet based applications and propose guidelines towards new development practices.
\end{abstract}

\section{INTRODUCTION}

The case of EC application development, presents for the IS profession new and unfamiliar challenges. Web based applications characteristics appear to challenge the appropriateness and relevance of methods and techniques followed for conventional systems. Moreover, the transition from a developed application to a system in use (and hence an Information System) presents user organisations and technology providers alike with unfamiliar complexities. For example in the analysis phase, developers face the pressure of user requirements in the absence of real users and within a very wide, open-ended solution space. The users of the Internet vary considerably demographically, making the analysis of their behaviour an extremely difficult task. Additionally technological capabilities such as hyperlinking within Internet applications makes their design complicated and difficult to standardise. Finally, testing and users feedback, have certain advantages in the new medium including speed and accuracy of user response. Therefore the body of knowledge concerning system development practice in the domain literature does not reflect the reality of such applications and does not address the requirements of EC projects. The need emerges for a reappraisal of system development theory and method in the light of practical, hands-on experience accumulated in the Industry through real life EC application development.

In this paper we demonstrate the merits of such an approach by abstracting from our practical project-based experience in EC development ${ }^{21}$. We discuss systematically the impact that specific characteristics of EC technology on systems development practice. In our approach we stress the transition from the traditional life cycle based conception of systems projects to the new reality created by electronic commerce.

\section{STANDARD ACTION GUIDELINES}

Despite criticisms on the relevance usefulness and effectiveness of IS development methodologies (Bransler \& Bodker 1993, Wynenkoop \& Russo 1995) these still feature prominently as a basis upon which developers should rely on within an IS project. Methodological guiding principles are summarised by livari (1991) along the following dimensions:

- IS planning, which refers to the development of the organisation-wide information system plan

- IS design, which is used to denote systems analysis and design activities involved in the development of individual application information systems

- IS implementation, is used in the sense of organisational implementation

- IS use, refers to the end-user's activities related to services provided by the system

- IS evolution, refers to enhancement type maintenance of the system

In this paper we adhere broadly to this terminology with some semantic changes regarding these guidelines. The IS planning for example, is examined not only within one organisation's limits, but includes also interorganisational systems. Additionally, the IS design will be separated in two phases: IS analysis and IS

21 Among the HELTRUN projects are the following:TAPPE - Telematics for Administrations: Public Procurement in Europe. An EU Telematics for Administration project (1997-1998) [17]; Newspaper Online - An Internet based Multimedia Information Retrieval System for a Greek newspaper (1997); [19,20]; COIN - Classifieds Over Internet, an interactive business to consumer web based application (1997. 1998); ACTIVE - Advertising and Commerce Through Internet in the context of Virtual Enterprise. An EU ESPRTT project for the establishment of a multi-agent electronic Retail marketplace (1998-) [18]; SYN - An intelligent electronic catalog system for the Athens Chamber of Commerce and Industry 
between chain stores and customers is a meaningful preparatory strategy.

\section{Analysis}

Problem Identification. The analysis phase in an IS project is concerned with determination of user requirements accompanied by all the other necessary negotiation processes that will enable the development of an application model (data, process, real time behaviour). A major difference between conventional and web based IS is the importance of market characteristics in defining application requirements. While in conventional IS, requirements are usually influenced by the employees who will be the direct users of the application, in web applications it is far more difficult to approach consumers-at-large who will be the ultimate end-users. Consumers usually present large discrepancies in demographic characteristics, market search patterns and buying behaviour. Moreover, the manner in which the proposed application will be integrated with existing systems needs to be addressed explicitly at two levels: The level of business processes and the level of internal information systems. For example orders received through the Internet need to be forwarded to the central processing system, while 'customer support' needs to be restructured to cater for Internet borned customers. This situation places emphasis on business modelling as part of systems analysis.

New conditions specification. Under the conditions discussed above analysis can only be effective as an interactive process driven by prototype development; this process is usually initially driven by technological capabilities of the application environment. Obviously a lot depends on the choice of technological platform and application tools used for the first prototype. However the real opportunity of web applications is the speed with which end user response concerning the initial prototype can be obtained. Subsequent rounds of prototype refinement can and should be informed by consumer related data, gathered through user monitoring and profiling.

Proposal for action. Analysis for web based development needs to draw on a variety of data resources: market data is needed to reveal structural characteristics that need to be reflected in the application functionality, and consumer-and product-related data that should serve as the basis for developing the application development model. Traditional data modelling methods such as entity relationship modelling have still a role to play in this stage. However it should be stressed that "analysis" for web applications can no longer be seen as an IS lifecycle stage; it is rather an iterative and interactive negotiation activity informed by user feedback obtained over the Internet.

\section{Design}

Problem Identification. There are two main areas of concern for designers of web applications (Balasubramanian et al 1995, Isakowitz et al 1995), the design of the application interface and the design of the underlying information management structure. Internet applications require a new conception of the role and functionality of the user interface. There are four areas of issues here. Firstly, the end user-consumer should have the feeling of contacting the physical entity (store, shopping mall, service provider, sales person) corresponding to the transaction being carried out. Secondly, the interface should reflect and exploit the necessary search and retrieval processes (concerning product characteristics etc) of the physical commercial environment. Moreover there is a need to promote the additional capabilities offer by the electronic medium (value added services such as customised services). Thirdly, the interface should offer clear guidance and support for the entire purchasing process; the particular problem here is that this process is made up by heterogeneous activities (searching through product features versus making an electronic payment). Lastly, the interface should offer capabilities to support related business process such as product promotion and advertisement. As far as the data design and management are concerned the requirements of web applications do not pose significant new challenges to designers and database design principles (Cutts 1991) can be used.

New conditions specification. Internet technology offers technical opportunities to address the above issues, in particular hyperlinking. This allows the easy navigation through the application's features and linking to other related commercial sites. Systems development methodologies (Balasubramanian et al 1995, Isakowitz et al 1995) specifically targeted to Internet applications offer concrete support for the design stage (e.g. automatic creation of web interface). Data design becomes a particularly important activity for Internet applications for two reasons. Firstly, because Internet applications are data driven in the sense that they are meant to reflect products, services, customers as well as the firm itself. Secondly, because through a solid data design the application developer has a greater grasp over the entire application as well as the capability to enhance and extend it in the future. Finally, integrated Electronic Commerce transactions require the seamless co-operation of a multitude of heterogeneous services (payment, shipping, dispatching). In many cases third party components are applicable, which offers opportunities for lowering the cost of application design and development but systems designers 
still need to create the links to the main application.

Proposal for action. Database design skills are a sine-qua-non within the context of web based applications. There is an opportunity here for systems professionals to capitalise on existing experience in totally new and innovative application fields. In essence the design of Internet applications sets off with the design of the database and processes with the embedding and the integration of new technologies such as web authoring tools or Java.

\section{Implementation}

Problem Identification. Conventionally the main challenges of systems implementation (i.e. "going live") have been the familiarisation of the users with a new environment and the migration from the old to the new system. Both these issues have very different profiles for Internet based applications. As far as users are concerned the onus lies on the system itself in capturing the attention, supporting the interaction and helping the user in cases of difficulty. With respect to migrating to a new way of work, business to consumer applications are usually complementary to traditional commerce operations. There are significant issues to be tackled in the restructuring of internal business processes (e.g. order processing, inventory and distribution management) to cater for the business environment. The integration of business processes creates new stakeholders for Internet applications, these are employees in other business functions who, although not directly involved with the application need to deal with business issues resulting from each operation.

New conditions specification. In addition to Internet applications under development there is the need to set up the transaction management environment for this application. Powerful middleware is available (Tennenbaum et al 1997, Evans \& Rogers 1997) but the important issue here is parameterisation. This process needs to take advantage of the potential of the technology while at the same time there is a need to provide also opportunities for improvement of existing business operations. For example, the monitoring of systems use and the collection of consumer related data might lead to the development of new marketing strategies. The monitoring of system use provides also data of the volume of users as well as of transactions created through the application, that are important for subsequent extension and exploitation of the application.

Proposal for action. Given the strong influence of technological capabilities offered within Electronic Commerce platforms, as well as by application development tools, a systems project in this area usually begins by the acquisition and installation of such a technological infrastructure and proceeds with the tailoring of each application component.

Use

Problem Identification. Business to Consumer Internet applications have a user community which is potentially the world-at-large. The very nature of these applications is significantly affected by the emerging mode of their use by consumers at large. Successful applications are those which incorporate feedback from end-users in their amended versions. This is an open-ended process, which requires the allocation of development effor on a continuous basis.

New conditions specification. The decision to use a Business to Consumer Internet application lies entirely on end-users. However, there are several factors that impact on that. With respect to the technological side of the system the necessary conditions for achieving successful system use can be summarised under the headings of user friendliness, simplicity, understadability, consistency and integrated support. However there are product/service as well as firm related characteristics that have a direct impact on system use in this case. Products or services offered through the Internet need to compete in terms of quality and cost on a global basis. Business processes of the firm need to be efficient and reliable in accordance to the "promises made" in the new system. In many cases system use is the first step in a process of sweeping changes across the organisation.

Proposal for action. A critical point in securing successful use of such applications is their careful promotion over electronic (e.g. advertisement in related sites) as well as over traditional means. In many cases such applications need to be based on a close collaboration between the providers of the products or services with the providers of technological capabilities, for example Internet providers as well as with business partners to whom particular processes are outsourced (e.g. distribution). In such cases system use may trigger a new round of system development activities aiming at the integration of systems across partners in the emerging virtual chain. 


\section{Systems development as systems evolution}

Business to Consumer Internet applications are being developed against the background of rapidly changing markets increasing sophistication of consumer behaviour, rapid technological change, significant legal and regulatory constrains and the current limited penetration of commercial Internet use in households (Cockburn and Wilson 1996). The terrain is changing fast. Many applications of today will not survive tomorrow without substantial rethinking and restructuring. In many cases current Business to Consumer applications are more significant as proof-of-concept vehicles (to explore the viability of Internet as a commerce medium) rather than as business entities in their own right. In this sense systems development does not end with a successful launching of the application over the Internet; but rather this is the point at which it takes off.

\section{CONCLUSIONS: IMPLICATIONS FOR RESEARCH AND PRACTICE}

Internet based applications are a prime example of systems projects that may be seen as beginning at the implementation stage. In a sense, the discussion on planning, analysis and design provided above, should be seen as the framework of issues to be considered at the outset of an Internet driven systems project. Given the strong influence of technological capabilities offered within Electronic Commerce, a systems project in this area usually begins by the acquisition and installation of such a technological infrastructure and proceeds with the tailoring and parameterisation of each component. This situation is not dissimilar to what takes place in other areas of systems development (e.g. integrated management support systems). Systems professionals and users alike need to adapt to the notion of projects evolving outside the systems lifecycle mentality. Such projects necessitate flexibility in time and resource allocation, emphasis on close collaboration between developers and clients, and ample contingencies.

The apparent challenges that this presents for the management of systems projects are mitigated by the capabilities that technology offers to developers for quick project turnover. The bottom line here is: negotiation, integration, feedback and evolutionary development. The adoption of an evolutionary mentality concerning the development of such systems is necessary for maintaining the momentum, preserving the experience gained by previous efforts and exploiting the impact of electronic presence in consumer markets. In creating a new body of knowledge for the information systems profession which will reflect the emerging conditions of development we suggest the following:

(i) the development of case study based templates of exemplar practices and the dissemination of such material over electronic means. The five dimensions of systems development reviewed in this paper may serve as a framework for structuring such material.

(ii) the fostering of closer collaboration across systems developers communities working on EC projects where ever such communities may be located: in academic and research institutions, in technology providers, in user organisations. A means for achieving this through the set up of networks of excellence using the model already practised in other areas of collaborative research, with special emphasis on industry specific EC requirements.

(iii) the development of closer links with existing fora already focused on awareness creation

(iv) the updating of academic and technical education curricula through the linking of traditional systems development competencies highlighted in this paper with specific requirements of this new type of systems applications.

\section{REFERENCES}

J. P. Bansler, K. Bodker, "A Reappraisal of Structured Analysis: Design in an Organisational Context", ACM Transactions on Information Systems, Vol. 11, No 2, April 1993, pp. 165-193

V. Balasubramanian, B. Ma Min, J. Yoo, "A systematic approach to designing a WWW application ", Communications of the ACM, August 1995, Vol. 38, No 8, pp. 47-48

R. Benjamin, and R. Wigand, "Electronic Markets and Virtual Value Chains on the Information Superhighway", Sloan Management Review, Winter 1995, pp. 62-72

C. Cockburn, T. Wilson, "Business Use of the World Wide Web", International Journal of Information Management, 1996, Vol. 16, No 2, pp. 83-102

Cutts G., "Structured analysis and design methodology", Alfred Waller Ltd. Publishers, 1991

T. Davenport, "Process Innovation", Harvard Business Press, 1994

W. Drakos, G. Doukidis, (1997), "Electronic Publishing over Internet: The Newspaper On line". 7th Mini Euro Conference on Electronic Commerce, Bruges, Belgium, March 1997.

W. Drakos, G. Doukidis, N. Papazafeiropoulou, (1997), "The Newspaper On-line Service: From a business to consumer application perspective". European Multimedia, Microprocessor Systems and Electronic Commerce Conference and Exposition (EMMSEC), Florence, Italy November 1997. 
European Workshop for Open Systems (EWOS) "Technical Guide on Electronic Commerce" EWOS ETG 066, Sep.1996

E. Evans, D. Rogers, "Using Java applets and CORBA for multi-user distributed applications", IEEE Internet Computing, May-June 1997, Vol. 1, No 3, pp 43-55

D.L.Hoffman, T.P. Novak, P.Chatterjee "Commercial scenarios for the Web:Opportunities and Challenges" Frontiers of Electronic Commerce Reading, MA:Addison Wesley 1996

J. Iivari, "A paradigmatic analysis of contemporary schools of IS development", European Journal of Information Systems, Vol. 1, No. 4, pp. 249-272, 1991

T. Isakowitz, E. Stohr, P. Balasubramanian, "RMM: A methodology for structured hypermedia design", Communications of the ACM, August 1995, Vol. 38, No 8, pp. 34-44

Kalakota R. and Whinston A., "Frontiers of electronic commerce", Addison-Wesley Publishing Company, inc 1996

C. Molenaar, "Interactive Marketing", Gower 1996

B. Pergioudakis, G. Doukidis and J. Pappas, "Defining an Architecture for Electronic Public Procurement in Europe". 10th International Bled Electronic Commerce Conference, Bled, Slovenia, June 1997.

J. M. Tennenbaum , T. S. Chowdhry, K. Hughes, "Eco System: An Internet Commerce Architecture", IEEE Computer, May 1997, Vol. 30, No 5, 48-55

G.Terpsidis, A.Moukas, B.Pergioudakis, G.Doukidis, P.Maes, "The potential of Electronic Commerce in reengineering consumer-retailer relationships through Intelligent Agents". European Multimedia, Microprocessor Systems and Electronic Commerce Conference and Exposition (EMMSEC), Florence, Italy November 1997.

S. Vinoski, "CORBA: Integrating diverse applications within distributed heterogeneous environments", IEEE Communications Magazine, February 1997, pp. 46-55

T. J. L. Wynenkoop, N. L. Russo, "Systems development methodologies: unanswered questions", Journal of Information Technology (10), 1995, pp. 65-73 